\title{
Heart Rate Reduction by Ivabradine Reduces Diastolic Dysfunction and Cardiac Fibrosis
}

\author{
David Busseuil $^{\mathrm{a}} \quad$ Yanfen Shi $^{\mathrm{a}} \quad$ Mélanie Mecteau $^{\mathrm{a}} \quad$ Geneviève Brand $^{\mathrm{a}}$ \\ Marc-Antoine Gillis ${ }^{a}$ Eric Thorin ${ }^{a} \quad$ Caroline Asselin ${ }^{a}$ Philippe Roméo ${ }^{a}$ \\ Tack Ki Leung $^{a} \quad$ Jean-Gilles Latour ${ }^{a} \quad$ Christine Des Rosiers ${ }^{a} \quad$ Muriel Bouly ${ }^{b}$ \\ Eric Rhéaume $^{a}$ Jean-Claude Tardifa \\ ${ }^{a}$ Research Center, Montreal Heart Institute, Université de Montréal, Montreal, Que., Canada; ${ }^{b}$ Institut de \\ Recherches Internationales Servier, Courbevoie, France
}

\section{Key Words}

Heart rate - Diastolic dysfunction - Collagen •

Echocardiography $\cdot$ Hypercholesterolemia

\begin{abstract}
Objectives: To determine if heart rate $(\mathrm{HR})$ reduction with ivabradine (IVA), a selective inhibitor of the pacemaker $I_{f}$ current, prevents cardiac dysfunction associated with dyslipidemia. Methods: New Zealand White rabbits received either a standard diet, a $0.5 \%$ cholesterol-enriched diet only (CD), or a $0.5 \%$ CD with IVA (17 mg/kg/day) for 12 weeks. HR, left ventricular (LV) systolic function, diastolic function and LV regional myocardial performance index (MPI) were studied using echocardiography. Histological analysis included cardiac interstitial fibrosis and collagen type I fibers. Plasma levels of angiotensin II and aldosterone were quantified by immunoassays. Results: IVA reduced HR by approximately $11 \%$. IVA improved MPI and attenuated LV diastolic dysfunction (DD) (92\% mild and $8 \%$ moderate DD with IVA vs. $54 \%$ mild and $46 \%$ moderate DD in CD group). IVA also reduced atrial fibrosis $(p=0.027)$, ventricular fibrosis $(p=0.0002)$ and ven-
\end{abstract}

tricular collagen type I ( $p=0.0042)$. IVA decreased plasma angiotensin II levels ( $p=0.042)$, and both angiotensin II and aldosterone levels were correlated with HR $(p=0.038$ and 0.008). Conclusion: Selective HR reduction with IVA reduces DD and cardiac fibrosis in hypercholesterolemic rabbits. These beneficial effects of IVA support testing pure HR reduction in patients with diastolic heart failure.

Copyright $\odot 2011$ S. Karger AG, Basel

\section{Introduction}

Increased resting heart rate (HR) is a risk factor for coronary artery disease, heart failure and mortality [1-3]. Studies have shown the benefits of lower HR on cardiovascular mortality and morbidity in different patient populations $[2,4,5]$. Although it was shown experimentally that stress-related $\beta$-adrenergic receptor-mediated increase in HR could promote vascular endothelial dysfunction and atherosclerosis in monkeys [6,7], the mechanisms linking cardiovascular diseases and HR remain largely unknown. An increase in HR mediated by $\beta$-ad-

\section{KARGER}

Fax +4161306 1234 E-Mail karger@karger.ch www.karger.com
(ㄷ) 2011 S. Karger AG, Basel

$0008-6312 / 10 / 1173-0234 \$ 26.00 / 0$

Accessible online at:

www.karger.com/crd
Jean-Claude Tardif, MD

Montreal Heart Institute

5000 Belanger Street, Montreal, QC H1T 1C8 (Canada)

Tel. +1 514376 3330, ext. 3612, Fax +1 5145932500

E-Mail jean-claude.tardif@icm-mhi.org 
renergic receptors following activation of the sympathetic nervous system is a common occurrence in heart failure [8], but this may be secondary to the cardiac dysfunction.

Ivabradine (IVA) reduces HR by a direct effect on the sinoatrial node [9]. It is an inhibitor of the $\mathrm{F}$ channel [10], which controls the electrical pacemaker activity in the sinoatrial node. IVA is deprived of inotropic and direct vasodilating effects [11-13]. Although the efficacy of IVA in the treatment of myocardial ischemia and angina has been shown [14], no study has directly evaluated its potential benefits on ventricular and atrial dysfunction in the context of dyslipidemia. Hypercholesterolemia has important implications for cardiac function. Elevated aortic afterload accelerated ventricular dysfunction in apolipoprotein E knockout mice [15] and 10 weeks of $0.5 \%$ cholesterol-enriched diet impairs systolic and diastolic function in rabbits [16]. Recently, we reported that moderate dyslipidemia led to cardiac dysfunction in addition to endothelial dysfunction in mice [17]. Therefore, in the present study, we have evaluated the effects of chronic HR reduction by IVA on diastolic dysfunction (DD) and cardiac fibrosis in cholesterol-fed rabbits.

\section{Methods}

\section{Animals and Experiments}

Animal care and procedures complied with the Canadian Council on Animal Care guidelines and were approved by the institutional ethics committee for animal research.

First, to evaluate the dose of IVA needed to induce a reduction in HR of approximately 15\%, rabbits were anesthetized (ketamine/midazolam 45/0.75 mg/kg), and electrocardiogram (ECG) radiofrequency transmitters TA10EAF40 (Data Sciences International, Arden Hills, Minn., USA) were placed subcutaneously. In 4 rabbits per group, ECG was monitored by telemetry by alternating data acquisition, 2 rabbits at a time for $24 \mathrm{~h}$. Signals were acquired simultaneously with IOX 2.3.7.5 (Emka Technologies, Paris, France). Recordings were analyzed and HRs calculated with ECG Auto 2.4.0.22 software (Emka Technologies). The dose of IVA was adjusted according to the dose-response curve obtained with the following results: reduction in $\mathrm{HR}$ was $1.8 \%$ with $1.0 \mathrm{mg} /$ $\mathrm{kg} /$ day of IVA, $3.4 \%$ with $5.0 \mathrm{mg} / \mathrm{kg} /$ day, $9.3 \%$ with $10 \mathrm{mg} / \mathrm{kg} / \mathrm{day}$, $17.1 \%$ with $20 \mathrm{mg} / \mathrm{kg} /$ day and $19.4 \%$ with $40 \mathrm{mg} / \mathrm{kg} / \mathrm{day}$.

Then, 32 male New Zealand White rabbits $(2.7-3.0 \mathrm{~kg}$, aged $12-13$ weeks) were assigned to receive either a standard diet (control group, $\mathrm{n}=6$ ), or a $0.5 \%$ cholesterol-enriched diet only (CD group, $\mathrm{n}=13)$, or a $0.5 \% \mathrm{CD}$ with IVA $(17 \mathrm{mg} / \mathrm{kg} / \mathrm{day}$, IVA group, $\mathrm{n}=13$ ) for 12 weeks. We initially aimed to induce a reduction in HR in the IVA group of approximately $15 \%$.

At 6 and 12 weeks, all rabbits were anesthetized (ketamine 30 $\mathrm{mg} / \mathrm{kg}$ and xylazine $5 \mathrm{mg} / \mathrm{kg}$ ) to monitor HR. All rabbits received similar controlled sedation. The dose of anesthetics was first administered according to the weight of each rabbit; moreover, the depth of anesthesia determined by evaluation of reflexes (corneal and toe pinch, leg withdrawal) and the pattern of respiration were monitored, and the dose of anesthetics was adjusted to reach a similar level of anesthesia in all animals. After 12 weeks (before sacrifice), cardiac function was studied by transthoracic echocardiography. HR and blood pressure were measured using a Millar catheter inserted in the right carotid artery. An arterial blood sample was collected for serum and plasma analyses, and cardiac tissues were harvested after sacrifice.

\section{Ivabradine}

IVA hydrochloride (S-16257-2) was provided by the Institut de Recherches Internationales Servier (Courbevoie, France) as a mono-hydrochloride salt. IVA was dissolved in water everyday and distributed in containers to the animals. The concentrations of IVA were regularly adjusted to rabbit body weight and water consumption.

\section{Serum and Plasma Analyses}

Total cholesterol, high-density lipoprotein (HDL) cholesterol, triglycerides, glucose and calcium levels were measured with an automated filter photometer system (Dimension RxL Max, Dade Behring, Deerfield, Ill., USA). Aldosterone levels were measured using a radio immunoassay kit (Coat-A-Count, DPC, Los Angeles, Calif., USA). Angiotensin II was measured using an enzyme immunoassay kit (SPI-Bio, Montigny-le-Bretonneux, France) following delipidation of the samples from the CD and IVA groups.

\section{Echocardiography}

A transthoracic echocardiographic study was performed before sacrifice, with rabbits being sedated by intramuscular injection of ketamine and midazolam (45 and $0.75 \mathrm{mg} / \mathrm{kg}$ ), using a phased-array probe 10S (4.5-11.5 MHz) and a Vivid 7 Dimension system (GE Healthcare Ultrasound, Horten, Norway).

Left ventricular (LV) M-mode spectrum was obtained in parasternal long-axis view to measure LV diameters at both end cardiac diastole (LVDd) and systole (LVDs). LV fractional shortening was calculated as (LVDd - LVDs)/LVDd $\times 100 \%$. The Teicholz method was employed to calculate LV volumes and LV ejection fraction.

Pulsed wave Doppler was used to evaluate transmitral flow and pulmonary venous flow in apical 4 -chamber view. Transmitral flow was used to measure the peak velocities during early filling (E) and atrial contraction (A) and to calculate the E/A ratio. Pulmonary venous flow was used to measure the systolic flow (S), diastolic flow (D) and reversed atrial flow (Ar). LV basal lateral peak systolic velocities $(\mathrm{Sm})$ and mitral annulus velocities during early filling (Em) and atrial filling (Am) were derived by tissue Doppler imaging. The time intervals from the end of Am to the beginning of Em (b), and from the beginning to the end of Sm (a) were also measured on lateral wall tissue Doppler imaging. LV myocardial performance index (MPI) was calculated by $(b-a) / a$ $\times 100 \%$. MPI combines LV systolic and diastolic properties together; it is a pure time interval index, non-geometric and independent of $\mathrm{HR}$, not requiring frequency-based normalization. Higher MPI implies impaired LV myocardial performance [18].

Left ventricular diastolic dysfunction (LVDD) was classified according to published criteria [19] (fig. 1a). To further evaluate LVDD, left atrium (LA) M-mode spectrum was obtained in parasternal long-axis view at the aortic valve level and LA dimensions were measured in both end cardiac diastole and systole. LA frac- 


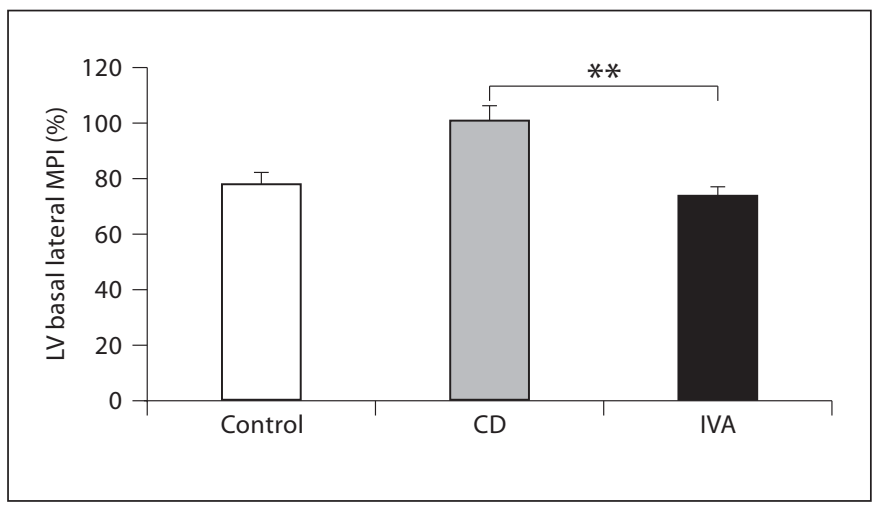

Fig. 1. LV basal lateral MPI. ${ }^{* *} \mathrm{p} \leq 0.01$.

tional shortening was calculated as (systolic dimension - diastolic dimension)/systolic dimension $\times 100 \%$. The average of 3 consecutive cardiac cycles was used for each measurement.

\section{Oxidative Stress Markers}

To evaluate the level of oxidative stress, we used a sensitive and specific gas chromatography-mass spectrometric assay of protein-bound 4-hydroxynonenal bound to thiol proteins (4HNE-P), a product of free radical-induced lipid peroxidation, as previously described [20]. Levels of reduced and oxidized glutathione were determined in $200 \mu \mathrm{l}$ of whole blood by capillary electrophoresis according to Serru et al. [21].

\section{Histology}

The LA and LV were dissected from the heart and immersion fixed in $10 \%$ buffered formalin at $4^{\circ} \mathrm{C}$ for $24 \mathrm{~h}$ and embedded in paraffin. Masson's Trichrome-stained sections were prepared for fibrosis area quantification. Deparaffinized ventricular sections were preincubated with an antigen unmasking solution (Vector Laboratories, Burlingame, Calif., USA), then immunostained with a goat polyclonal antibody against collagen type I (1:10 dilution; Millipore, Billerica, Mass., USA). Species-appropriate biotinylated secondary antibody was applied, followed by streptavidin-horseradish peroxidase complex, visualized with amino-9ethylcarbazol and counterstained by Mayer's hematoxylin.

The acquisition of 5 histologic images per section was based on complete atrial and ventricular slices, considering every level of tissue wall, without any selection of a specific zone and without any information on the rabbit study group. Images were acquired at $20 \times$ magnification using a light microscope (BX41, Olympus, Tokyo, Japan) connected to a digital video camera (Qcolor3, Olympus). Automatic computer-based analysis was performed with the same color threshold for all specimens. Data were expressed as percent stained area in the region of analysis.

\section{Statistical Analysis}

Data are presented as the mean \pm standard error of the mean for continuous variables and as frequencies and percentages for categorical variables. DD classification was compared across groups using the $\chi^{2}$ test. Lipid, circulating, echocardiographic and histologic parameters were compared across groups (control group, CD group and IVA group) using the one-way ANOVA model followed by pairwise comparisons if the global F test was significant at the 0.05 level. For LA fractional shortening, the contrast under the ANOVA model for comparison between the CD and IVA groups was evaluated as an exploratory analysis if the global $\mathrm{F}$ test was $\leq 0.10$. Parameters only measured for $\mathrm{CD}$ and IVA groups were compared between them using Student's t test. Pearson correlations were performed to investigate the relationships between HR and both aldosterone and angiotensin II. All analyses were done with SAS version 9.1 (SAS Institute Inc., Cary, N.C., USA) and conducted at the 0.05 significance level.

\section{Results}

\section{Lipid Levels, HR and Blood Pressure Lipids}

No significant difference between IVA and CD groups was observed at the end of the study for serum levels of total cholesterol, high-density lipoprotein cholesterol, triglycerides, glycemia and calcemia (table 1).

\section{Heart Rate}

Using telemetric measures in the preliminary study, the dose of IVA was set to $17 \mathrm{mg} / \mathrm{kg} /$ day to reach an expected decrease in HR of 15\% (see Methods). At 6 weeks of treatment in the main study, echocardiographic measurements under anesthesia showed a mean decrease in HR of $11.4 \%$ in the IVA group compared with the CD group (220 \pm 7 vs. $245 \pm 9 \mathrm{bpm} ; \mathrm{p}=0.035)$. At 12 weeks, $\mathrm{HR}$ was $270 \pm 19,218 \pm 9$ and $200 \pm 8 \mathrm{bpm}$ in the control, CD and IVA group, respectively $(\mathrm{p}=0.0003$ for IVA vs. controls).

\section{Blood Pressure}

Mean blood pressure was not significantly different among the control, CD and IVA groups (table 2).

\section{Cardiac Function}

LV Myocardial Performance Index

LV basal lateral MPI was 78.1 $\pm 4.3,100.7 \pm 5.4$ and $73.4 \pm 3.7 \%$ in the control, CD and IVA group, respectively ( $p=0.005$ for IVA vs. CD; $p=$ not significant for IVA vs. controls; fig. 1), indicating that IVA prevented the detrimental effects of the cholesterol diet on LV myocardial performance.

\section{Systolic Function}

LV fractional shortening and LV ejection fraction were similar in the control, CD and IVA groups (table 2), indicating that the LV global systolic function was well preserved in all cholesterol-enriched diet rabbits. 
Table 1. Blood analyses

\begin{tabular}{lccc}
\hline & Control $(\mathrm{n}=6)$ & CD $(\mathrm{n}=13)$ & IVA $(\mathrm{n}=13)$ \\
\hline Total cholesterol, mmol/l & $0.6 \pm 0.1$ & $20.8 \pm 1.6^{\star *}$ & $20.3 \pm 2.5^{\star *}$ \\
HDL cholesterol, mmol/l & $0.3 \pm 0.0$ & $0.8 \pm 0.0^{\star *}$ & $0.8 \pm 0.1^{\star *}$ \\
Triglycerides, mmol/l & $1.2 \pm 0.2$ & $1.6 \pm 0.3$ & $1.4 \pm 0.2$ \\
Glycemia, mmol/l & $11.3 \pm 0.8$ & $9.9 \pm 0.5$ & $9.9 \pm 0.6$ \\
Calcemia, mmol/l & $3.1 \pm 0.0$ & $2.9 \pm 0.0$ & $3.1 \pm 0.1$ \\
Angiotensin II, AU/ml & - & $16.7 \pm 4.8$ & $5.5 \pm 1.2^{\star * *}$ \\
Aldosterone, pmol/l & $386 \pm 79$ & $248 \pm 44$ & $163 \pm 26^{\star}$ \\
4HNE-P, pmol/mg protein & $0.51 \pm 0.06$ & $2.72 \pm 0.35^{\star *}$ & $2.60 \pm 0.40^{\star *}$ \\
GSH/GSSG & $8.7 \pm 1.6$ & $15.8 \pm 1.4$ & $22.0 \pm 3.3^{*}$ \\
\hline
\end{tabular}

$\mathrm{HDL}=$ High-density lipoprotein; GSH = reduced glutathione; GSSG = oxidized glutathione. ${ }^{*} \mathrm{p} \leq 0.01$ versus control; ${ }^{* *} \mathrm{p} \leq 0.001$ versus control; ${ }^{* *} \mathrm{p} \leq 0.05$ versus $\mathrm{CD}$.

Table 2. Echocardiographic and hemodynamic results after 12 weeks of treatment

\begin{tabular}{llll}
\hline & Control $(\mathrm{n}=6)$ & CD $(\mathrm{n}=13)$ & IVA $(\mathrm{n}=13)$ \\
\hline LV fractional shortening, \% & $38.1 \pm 1.1$ & $40.2 \pm 1.4$ & $43.1 \pm 1.2$ \\
LV ejection fraction, \% & $72.2 \pm 1.4$ & $74.2 \pm 1.6$ & $77.5 \pm 1.3$ \\
Pulmonary venous S wave, cm/s & $47.3 \pm 4.1$ & $33.9 \pm 2.9^{\mathrm{a}}$ & $41.1 \pm 2.4^{\mathrm{b}}$ \\
Pulmonary venous D wave, cm/s & $33.0 \pm 2.0$ & $32.6 \pm 1.7$ & $35.1 \pm 2.3$ \\
Pulmonary venous Ar, cm/s & $23.8 \pm 2.9$ & $20.5 \pm 2.0$ & $19.8 \pm 1.1$ \\
Mitral E/A ratio & $1.34 \pm 0.28$ & $1.17 \pm 0.12$ & $1.16 \pm 0.16$ \\
LA fractional shortening, \% & $26.6 \pm 1.6$ & $23.3 \pm 1.3$ & $27.6 \pm 1.5^{\mathrm{e}}$ \\
LA systolic dimension, cm & $0.93 \pm 0.05$ & $1.05 \pm 0.04$ & $1.01 \pm 0.03$ \\
LA diastolic dimension, cm & $0.68 \pm 0.04$ & $0.81 \pm 0.04$ & $0.73 \pm 0.04$ \\
LV basal lateral MPI & $78.1 \pm 4.3$ & $100.7 \pm 5.4$ & $73.4 \pm 3.7^{\mathrm{c}}$ \\
Systolic blood pressure, mm Hg & $70.2 \pm 3.3$ & $73.6 \pm 2.3$ & $79.5 \pm 1.8^{\mathrm{d}}$ \\
Diastolic blood pressure, mm Hg & $50.2 \pm 3.2$ & $56.7 \pm 2.2$ & $57.3 \pm 1.3$ \\
Mean blood pressure, mm Hg & $56.8 \pm 3.1$ & $63.4 \pm 2.3$ & $64.7 \pm 1.4$ \\
\hline
\end{tabular}

$\mathrm{S}=$ Systolic flow; $\mathrm{D}=$ diastolic flow; $\mathrm{Ar}=$ reversed atrial flow; $\mathrm{E}=$ peak velocity in early filling; $\mathrm{A}=$ peak velocity during atrial contraction. ${ }^{a} \mathrm{p} \leq 0.01$ versus control; ${ }^{\mathrm{b}} \mathrm{p}=0.06$ versus $\mathrm{CD} ;{ }^{\mathrm{c}} \mathrm{p} \leq 0.01$ versus $\mathrm{CD} ;{ }^{\mathrm{d}} \mathrm{p} \leq 0.05$ versus control; ${ }^{\mathrm{e}} \mathrm{p}=0.038$ versus $\mathrm{CD}$ (for LA fractional shortening, exploratory analysis given $\mathrm{p}=0.10$ for global $\mathrm{F}$ test).

\section{Diastolic Function}

Improved MPI with unchanged LV systolic function suggested that IVA improved diastolic function. Indeed, LV diastolic filling patterns (fig. 2a) were distributed differently among groups $(\mathrm{p}=0.0003)$. $\mathrm{DD}$, observed in the CD group, was attenuated by IVA $(92.3 \%$ of mild DD and $7.7 \%$ of moderate DD vs. $53.8 \%$ of mild DD and $46.2 \%$ of moderate DD for CD rabbits) (fig. 2b). Moderate DD occurred significantly more frequently in the CD group than in the IVA group ( $p=0.027$; fig. $2 b)$. Pulmonary venous $S$ wave was higher in controls than in the $\mathrm{CD}$ group $(\mathrm{p}=0.008)$, as well as in rabbits treated with IVA versus $\mathrm{CD}(\mathrm{p}=0.064)$ (table 2). LA fractional shortening was $26.6 \pm 1.6 \%$ in the control group (global; $\mathrm{p}=0.10$ ), 27.6 $\pm 1.5 \%$ in the IVA group ( $\mathrm{p}=0.038$ vs. CD) and $23.3 \pm$ $1.3 \%$ in the CD group (table 2).

\section{Histology - LA and LV Interstitial Fibrosis and LV} Collagen Type I

IVA treatment significantly reduced the extent of LA interstitial fibrosis (blue staining; fig. 3a) compared with the CD group $[16.5 \pm 1.1 \%(\mathrm{n}=7)$ vs. $20.9 \pm 1.4 \%(\mathrm{n}=$ 


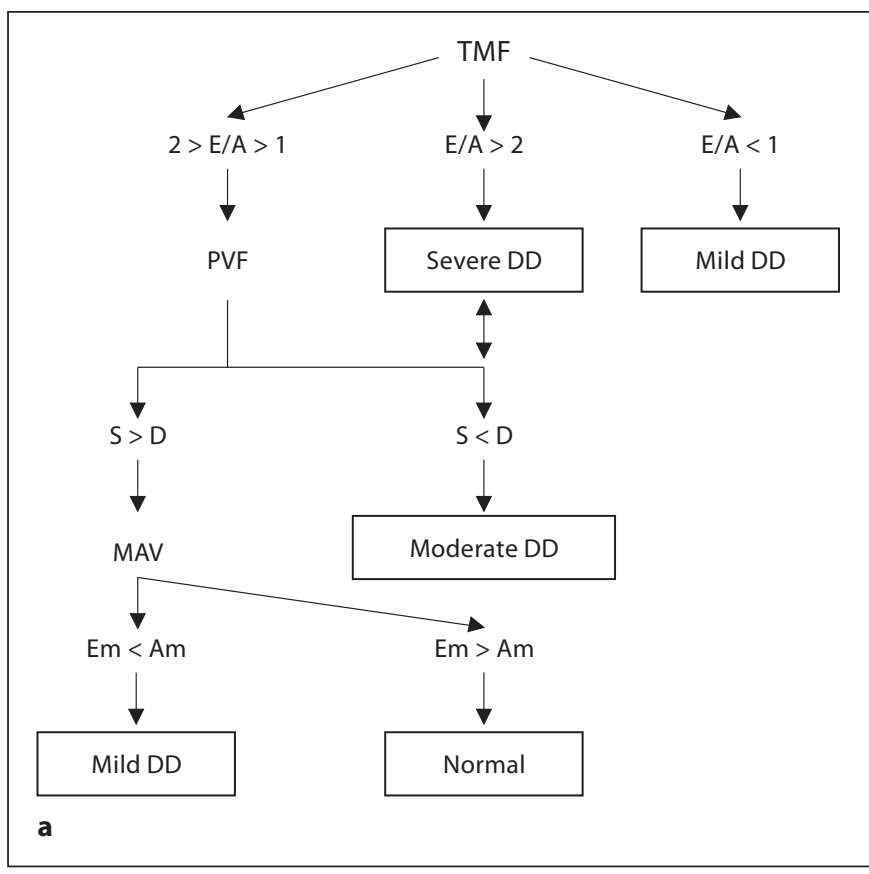

Fig. 2. LVDD severity. a LVDD classification: mild LVDD was defined by $\mathrm{E} / \mathrm{A}<1$, or $1<\mathrm{E} / \mathrm{A}<2$, with $\mathrm{S} / \mathrm{D}>1$ and $\mathrm{Em}<\mathrm{Am}$. Moderate LVDD was considered present when $2>\mathrm{E} / \mathrm{A}>1$ with $\mathrm{S} / \mathrm{D}<1$. Severe LVDD was diagnosed when $\mathrm{E} / \mathrm{A}>2$ with $\mathrm{S} / \mathrm{D}<1$. TMF = Transmitral flow; $\mathrm{E}=$ peak velocity in early filling; $\mathrm{A}=$ peak velocity during atrial contraction; $\mathrm{PVF}=$ pulmonary venous

9); $p=0.027$ ] but did not normalize it (fig. 3b). In contrast, IVA treatment reduced LV interstitial fibrosis (fig. 3a) to the almost normal value of $1.2 \pm 0.2 \%$, compared with $5.8 \pm 0.9 \%$ in the CD group ( $\mathrm{p}=0.0002$; fig. $3 \mathrm{~b})$. Ventricular fibrosis was $0.5 \pm 0.1 \%$ in the control rabbits ( $\mathrm{p}=0.602$ for IVA vs. control rabbits; $\mathrm{p}=0.0008$ for $\mathrm{CD}$ vs. control rabbits).

IVA also reduced the extent of LV collagen type I to 2.7 $\pm 0.3 \%$, compared with $9.6 \pm 1.6 \%$ in the CD group $(\mathrm{p}=0.0042)$. Collagen type I was $4.8 \pm 0.7 \%$ in the normal control rabbits ( $\mathrm{p}=$ not significant for IVA vs. control rabbits; $\mathrm{p}=0.0248$ for $\mathrm{CD}$ vs. control rabbits).

\section{Circulating Markers}

Plasma Levels of Angiotensin II and Aldosterone

Plasma levels of angiotensin II, which were measured in the hypercholesterolemia groups only (table 1), were reduced with IVA compared with the CD group ( $\mathrm{p}=$ 0.042) (fig. 4a). Plasma angiotensin II was significantly correlated with HR at 12 weeks for pooled CD and IVA animals $(r=0.37, p=0.038)$. Aldosterone levels were decreased in the IVA versus the control group ( $p=0.004$;

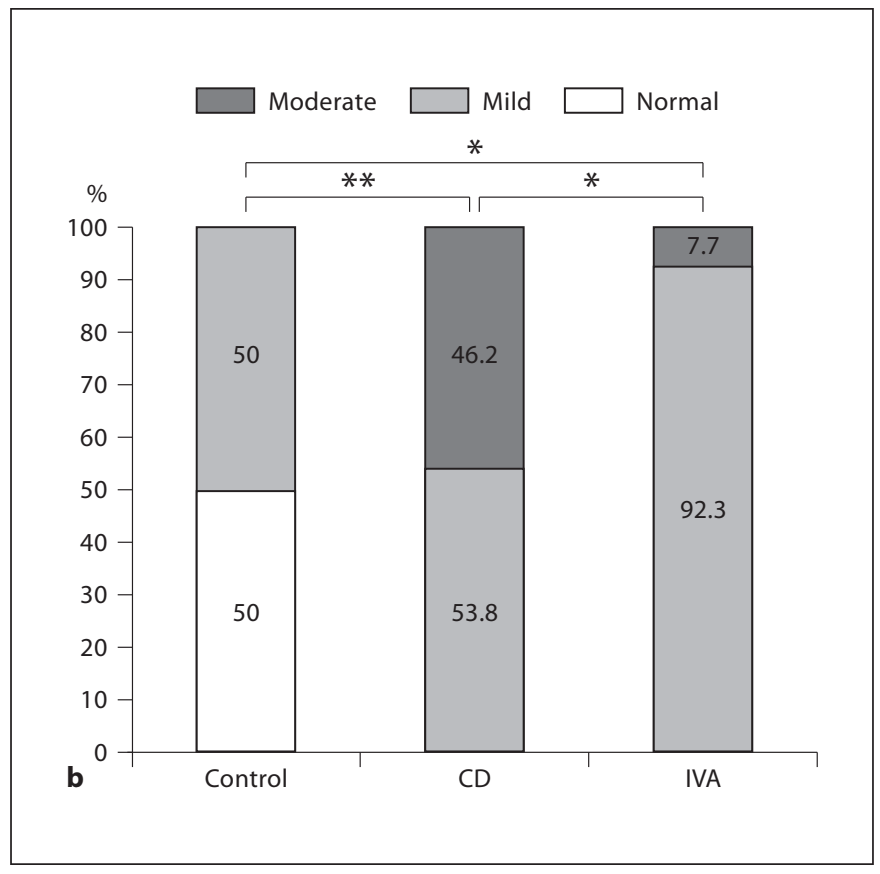

flow; $\mathrm{S}=$ systolic flow $\mathrm{D}=$ diastolic flow; $\mathrm{MAV}=$ mitral annulus velocity; Em = mitral annulus velocity during early filling; $\mathrm{Am}=$ mitral annulus velocity during atrial contraction. b Distribution of LVDD severity in control, CD and IVA groups at 12 weeks after treatment. ${ }^{*} \mathrm{p} \leq 0.05,{ }^{* *} \mathrm{p} \leq 0.01$.

table 1) and tended to be lower in the IVA versus the CD group ( $\mathrm{p}=0.142$ ). Plasma levels of aldosterone were also significantly correlated with $\mathrm{HR}$ for the pooled study animals ( $r=0.46, p=0.008$; fig. $4 b)$.

\section{Oxidative Stress}

Values for the lipid peroxidation marker 4HNE-P were higher in both hypercholesterolemic groups compared with controls (vs. CD, $\mathrm{p}<0.0001$; vs. IVA, $\mathrm{p}=0.0002$; table 1). Reduced glutathione/oxidized glutathione ratio in blood, a marker of the thiol redox status, was significantly higher in IVA compared with the control group ( $\mathrm{p}=0.004$; table 1 ), but not with the $\mathrm{CD}$ group, nor was the CD group different from controls.

\section{Discussion}

This study demonstrates that selective HR reduction with IVA prevented the detrimental effects of hypercholesterolemia on LV myocardial performance. IVA attenuated LV diastolic dysfunction, reduced LA and LV struc- 

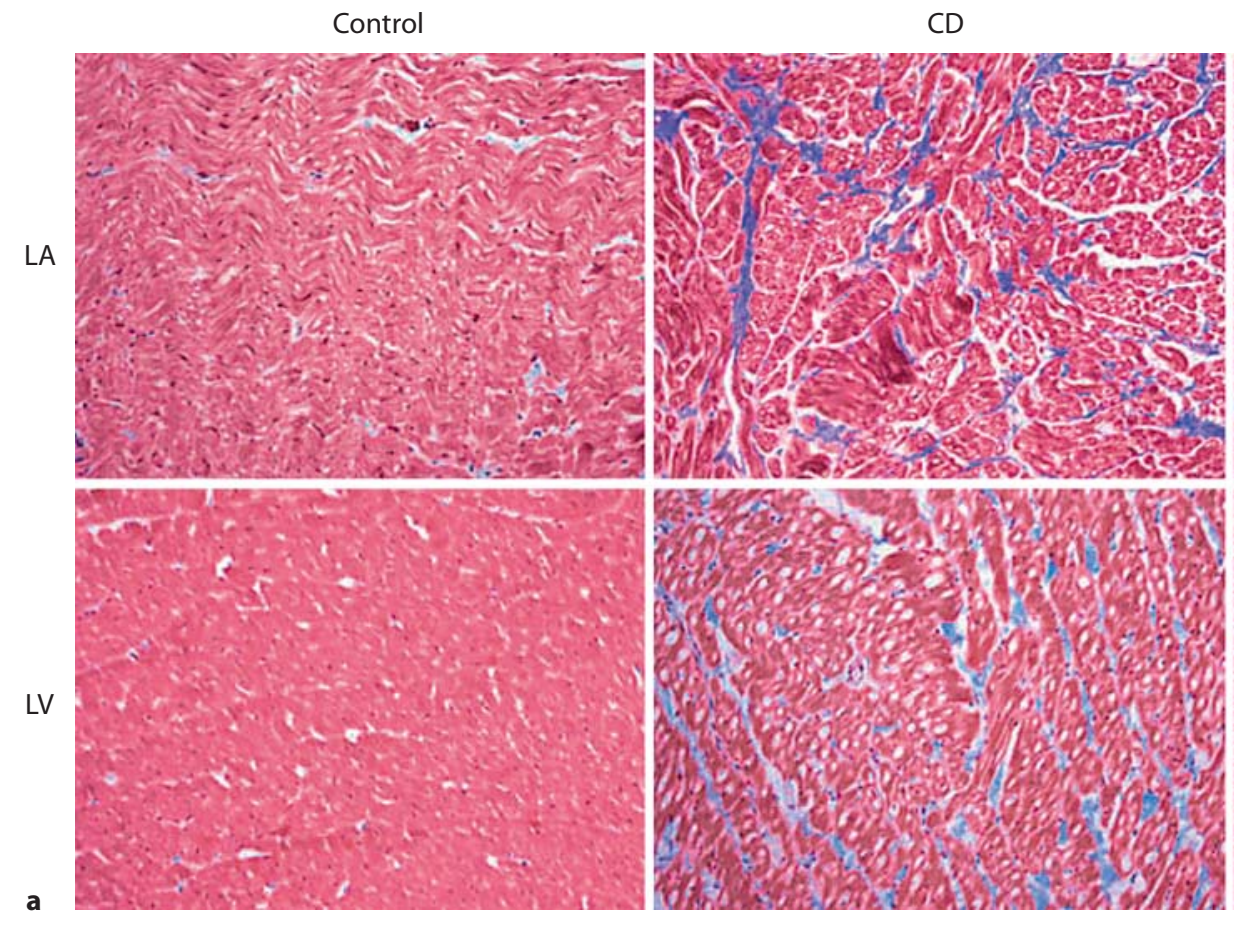

IVA

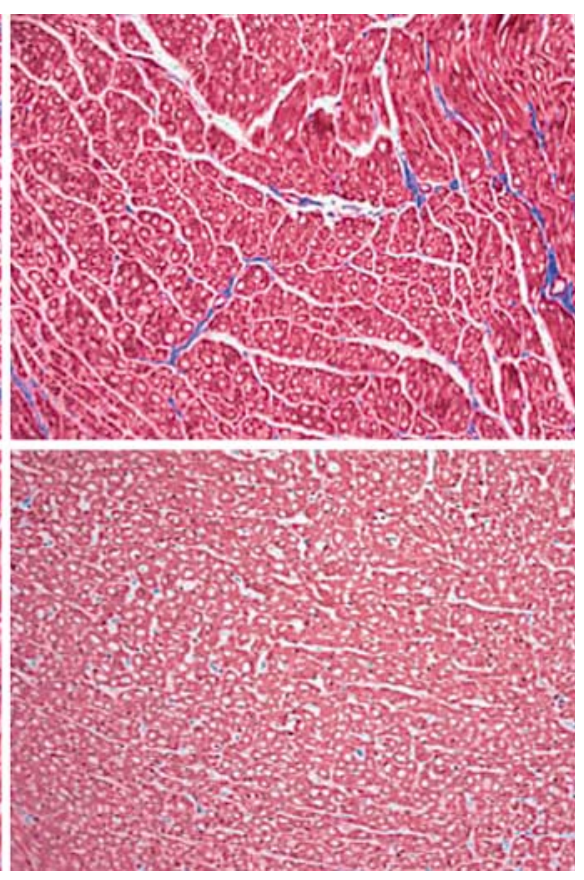

Fig. 3. Histology of LA and LV interstitial fibrosis. a Masson's Trichrome staining of LA and LV sections (images acquired at $20 \times$ magnification for each panel). Fibrosis is stained in blue; atrial and ventricular muscle is stained in pink. b Quantification by digital image analysis of interstitial fibrosis in the LA and LV sections of control, CD and IVA groups, expressed as the percentage of stained area in the region of analysis. ${ }^{*} \mathrm{p} \leq 0.05,{ }^{* * *} \mathrm{p} \leq 0.001$.

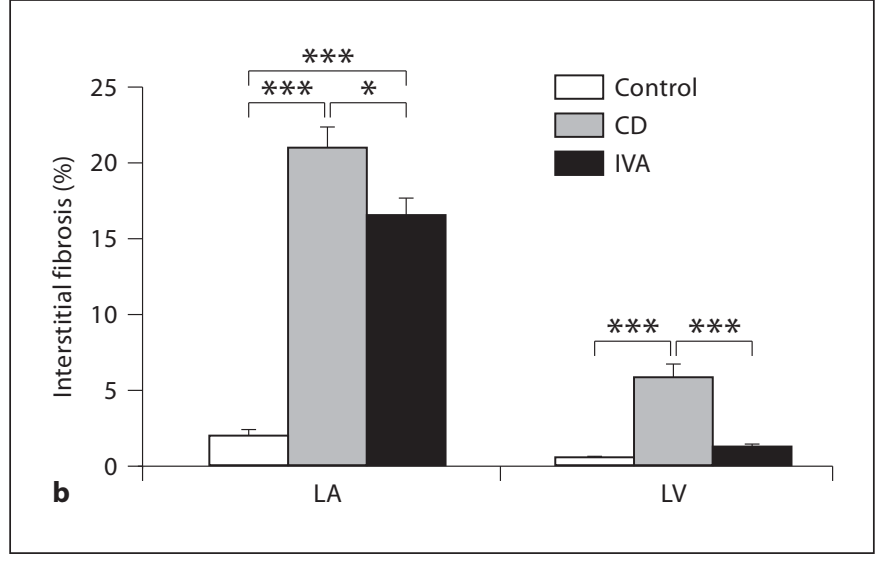

dysfunction, the significant improvement in LV MPI reflects improved LV diastolic function with IVA. In our current study, chronic reduction in HR by IVA indeed limited LVDD in dyslipidemic rabbits. Our observations are in agreement with previous results reported in dyslipidemic mice [17] and in a rat model of heart failure induced by coronary artery ligation [24]. We found that DD of moderate severity developed in dyslipidemic rabbits, and this was significantly attenuated by the treatment with IVA. These echocardiographic results are concordant with the large reduction in ventricular fibrosis and collagen type I with IVA observed on histology. There- 

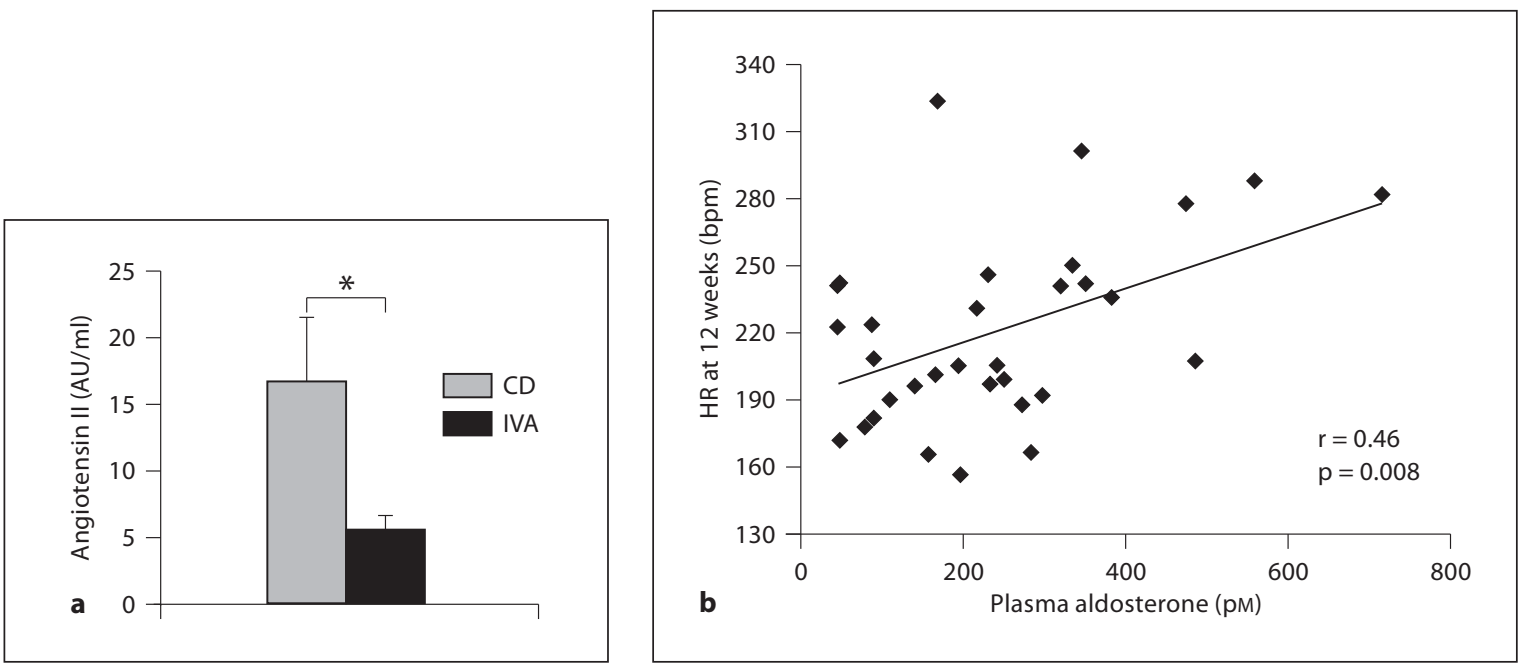

Fig. 4. a Plasma angiotensin II levels in CD and IVA groups. ${ }^{*} \mathrm{p} \leq 0.05$. AU = Arbitrary units. b Correlation between HR at 12 weeks and plasma aldosterone levels in animals from pooled control, CD and IVA groups.

fore, there is a significant benefit of selectively lowering HR in the presence of diastolic dysfunction.

We have previously described the prognostic implications of DD detected by echocardiography [25]. We have also previously reported the effects of anesthesia and surgery on diastolic function [26], but all the animals in the present study were submitted to the same degree of anesthesia. The systolic component of the pulmonary venous flow (S wave) has been shown to correlate well with LV filling pressure and with its change over time [27]. Therefore, our assessment of DD was based on the classification recommended by the American Society of Echocardiography, where the pulmonary venous systolic/diastolic flow ratio plays a key role [19].

Elevated concentrations of angiotensin II associated with heart failure have been shown to promote atrial fibrosis [28]. In our dyslipidemia-induced DD model, IVA decreased circulating angiotensin II levels, decreased fibrosis of both the LA and LV walls and decreased collagen type I in LV walls. Reduction in perivascular collagen in ventricular myocardium has been reported with IVA in middle-aged postmyocardial infarction rats [29]. Given the importance of atrial fibrosis in the pathophysiology of atrial fibrillation [30], our observation of reduced atrial and ventricular fibrosis induced by IVA in the setting of dyslipidemia-induced DD potentially opens a new area of investigation for the prevention of this common arrhythmia. Moreover, plasma levels of angiotensin II and aldosterone were positively correlated with HR dur- ing treatment in our study. These observations are particularly interesting in light of the recent finding that the increased rates of mortality, vascular events and heart failure associated with the use of the lipid-modulating agent torcetrapib were due at least in part to an increase in aldosterone levels [31].

In this hypercholesterolemic rabbit model, the improvement in diastolic function associated with selective HR reduction was associated with IVA-induced reduction in ventricular fibrosis and collagen type I. The improvement in cardiac function and fibrosis [32] could be dependent on the combined reduced levels of angiotensin and aldosterone, which act in synergy during cardiac remodeling [33]. The favorable effects on diastolic function may also in part have been due to IVA-induced increase in diastolic (ventricular filling) time, as described in previous experimental studies [17, 24]; improved myocardial perfusion may also have contributed to the beneficial effects of IVA due to the lengthening of diastole and reduced perivascular collagen [12, 29].

The benefits conferred by IVA were observed independent of effects on hypercholesterolemia and associated oxidative stress. In fact, as expected, circulating levels of cholesterol and of the lipid peroxidation marker 4HNE-P were increased in rabbits fed the hypercholesterolemic diet [34], but were not affected by IVA treatment. However, the possibility that IVA attenuates oxidative stress at the tissue level (i.e. myocardium) cannot be excluded. Indeed, in a previous study conducted in ApoE-/- mice fed 
with a cholesterol-rich diet for 6 weeks, IVA-induced HR reduction was associated with decreases in NADPH oxidase activity, superoxide production and lipid peroxidation in the vascular wall [35].

\section{Conclusion}

Selective HR reduction with IVA in hypercholesterolemic rabbits attenuates LVDD, reduces LA and LV fibrosis and reduces LV collagen type I. Circulating angiotensin II and aldosterone levels were lowered with IVA and correlated with HR. These beneficial effects of IVA support the evaluation of pure HR reduction in patients with diastolic heart failure.

\section{Acknowledgments}

The authors gratefully acknowledge the statistical analysis by Marieve Cossette, MSc, and Marie-Claude Guertin, $\mathrm{PhD}$, at the Montreal Heart Institute Coordinating Center.

\section{Conflicts of Interest}

Dr. Tardif holds the Université de Montréal research chair in atherosclerosis. Dr. Tardif has received honoraria from Servier. Dr. Busseuil holds a research fellowship award of the Heart and Stroke Foundation of Canada.

\section{References}

1 Kannel WB: Heart rate and cardiovascular mortality. The Framingham Study. Am Heart J 1987;113:1489-1494.

-2 Diaz A, Bourassa MG, Guertin MC, Tardif JC: Long-term prognostic value of resting heart rate in patients with suspected or proven coronary artery disease. Eur Heart J 2005; 26:967-974

3 Fox K, Ford I, Steg PG, Tendera M, Robertson M, Ferrari R, BEAUTIFUL investigators: Heart rate as a prognostic risk factor in patients with coronary artery disease and leftventricular systolic dysfunction (BEAUTIFUL): a subgroup analysis of a randomised controlled trial. Lancet 2008;372:817-821.

$\checkmark 4$ Palatini P, Julius S: Elevated heart rate: a major risk factor for cardiovascular disease. Clin Exp Hypertens 2004;26:637-644

5 Gillman MW, Kannel WB, Belanger A, D'Agostino RB: Influence of heart rate on mortality among persons with hypertension: the Framingham Study. Am Heart J 1993; 125:1148-1154.

-6 Kaplan JR, Manuck SB, Clarkson TB: The influence of heart rate on coronary artery atherosclerosis. J Cardiovasc Pharmacol 1987; 10(suppl 2):S100-S102.

-7 Strawn WB, Bondjers G, Kaplan JR, Manuck SB, Schwenke DC, Hansson GK, Shively CA, Clarkson TB: Endothelial dysfunction in response to psychosocial stress in monkeys. Circ Res 1991;68:1270-1279.

8 Cohn JN: Abnormalities of peripheral sympathetic nervous system control in congestive heart failure. Circulation 1990;82:159167.

9 Ragueneau I, Laveille C, Jochemsen R, Resplandy G, Funck-Brentano C, Jaillon P: Pharmacokinetic-pharmacodynamic modeling of the effects of ivabradine, a direct sinus node inhibitor, on heart rate in healthy volunteers. Clin Pharmacol Ther 1998;64:192-203.
10 Bucchi A, Baruscotti M, DiFrancesco D: Current-dependent block of rabbit sino-atrial node I(f) channels by ivabradine. J Gen Physiol 2002;120:1-13.

11 Gardiner SM, Kemp PA, March JE, Bennett $\mathrm{T}$ : Acute and chronic cardiac and regional haemodynamic effects of the novel bradycardic agent, S16257, in conscious rats. Br J Pharmacol 1995;115:579-586.

12 Simon L, Ghaleh B, Puybasset L, Giudicelli JF, Berdeaux A: Coronary and hemodynamic effects of S16257, a new bradycardic agent, in resting and exercising conscious dogs. Pharmacol Exp Ther 1995;275:659-666.

13 Colin P, Ghaleh B, Hittinger L, Monnet X, Slama M, Giudicelli JF, Berdeaux A: Differential effects of heart rate reduction and beta-blockade on left ventricular relaxation during exercise. Am J Physiol Heart Circ Physiol 2002;282:H672-H679.

14 Borer JS, Fox K, Jaillon P, Lerebours G: Antianginal and anti-ischemic effects of ivabradine, an I(f) inhibitor, in stable angina: a randomized, double-blind, multicentered, placebo-controlled trial. Circulation 2003;107: 817-823.

15 Wu JH, Hagaman J, Kim S, Reddick RL, Maeda N: Aortic constriction exacerbates atherosclerosis and induces cardiac dysfunction in mice lacking apolipoprotein E. Arterioscler Thromb Vasc Biol 2002;22:469-475.

$>16$ Huang Y, Walker KE, Hanley F, Narula J, Houser SR, Tulenko TN: Cardiac systolic and diastolic dysfunction after a cholesterolrich diet. Circulation 2004;109:97-102.

$\checkmark 17$ Drouin A, Gendron ME, Thorin E, Gillis MA, Mahlberg-Gaudin F, Tardif JC: Chronic heart rate reduction by ivabradine prevents endothelial dysfunction in dyslipidaemic mice. Br J Pharmacol 2008;154:749-757.
18 Broberg CS, Pantely GA, Barber BJ: Validation of the myocardial performance index by echocardiography in mice: a noninvasive measure of left ventricular function. J Am Soc Echocardiogr 2003; 16:814-823.

19 Khouri SJ, Maly GT, Suh DD, Walsh TE: A practical approach to the echocardiographic evaluation of diastolic function. J Am Soc Echocardiogr 2004;17:290-297.

20 Asselin C, Bouchard B, Tardif JC, Des Rosiers C: Circulating 4-hydroxynonenal-protein thioether adducts assessed by gas chromatography-mass spectrometry are increased with disease progression and aging in spontaneously hypertensive rats. Free Radic Biol Med 2006;41:97-105.

-21 Serru V, Baudin B, Ziegler F, David JP, Cals MJ, Vaubourdolle M, Mario N: Quantification of reduced and oxidized glutathione in whole blood samples by capillary electrophoresis. Clin Chem 2001;47:1321-1324.

22 Cuniberti LA, Stutzbach PG, Guevara E, Yannarelli GG, Laguens RP, Favaloro RR: Development of mild aortic valve stenosis in a rabbit model of hypertension. J Am Coll Cardiol 2006;47:2303-2309.

23 Nagueh SF, Chen S, Patel R, Tsybouleva N, Lutucuta S, Kopelen HA, Zoghbi WA, Quinones MA, Roberts R, Marian AJ: Evolution of expression of cardiac phenotypes over a 4-year period in the beta-myosin heavy chain-Q403 transgenic rabbit model of human hypertrophic cardiomyopathy. J Mol Cell Cardiol 2004;36:663-673.

24 Mulder P, Barbier S, Chagraoui A, Richard V, Henry JP, Lallemand F, Renet S, Lerebours G, Mahlberg-Gaudin F, Thuillez C: Longterm heart rate reduction induced by the selective I(f) current inhibitor ivabradine improves left ventricular function and intrinsic myocardial structure in congestive heart failure. Circulation 2004;109:1674-1679. 
-25 Denault AY, Couture P, Tardif JC: Left and right ventricular diastolic dysfunction as predictors of difficult separation from cardiopulmonary bypass. Can J Anaesth 2006; 53:1020-1029.

-26 Shi Y, Denault AY, Couture P, Butnaru A, Carrier M, Tardif JC: Biventricular diastolic filling patterns after coronary artery bypass graft surgery. J Thorac Cardiovasc Surg 2006;131:1080-1086.

$\checkmark 27$ Kuecherer HF: Estimation of mean left atrial pressure from transesophageal pulsed Doppler echocardiography of pulmonary venous flow. Circulation 1990;82:1127-1139.

-28 Cha YM, Redfield MM, Shen WK, Gersh BJ: Atrial fibrillation and ventricular dysfunction: a vicious electromechanical cycle. Circulation 2004; 109:2839-2843.
29 Dedkov EI, Zheng W, Christensen LP, Weiss RM, Mahlberg-Gaudin F, Tomanek RJ: Preservation of coronary reserve by ivabradineinduced reduction in heart rate in infarcted rats is associated with decrease in perivascular collagen. Am J Physiol Heart Circ Physiol 2007;293:H590-H598.

30 Li D, Fareh S, Leung TK, Nattel S: Promotion of atrial fibrillation by heart failure in dogs: atrial remodeling of a different sort. Circulation 1999;100:87-95.

31 Barter PJ, Caulfield M, Eriksson M, Grundy SM, Kastelein JJ, Komajda M, Lopez-Sendon J, Mosca L, Tardif JC, Waters DD, Shear CL, Revkin JH, Buhr KA, Fisher MR, Tall AR, Brewer B, ILLUMINATE investigators: Effects of torcetrapib in patients at high risk for coronary events. N Engl J Med 2007;357: 2109-2122.

-32 Montezano AC, Touyz RM: Networking between systemic angiotensin II and cardiac mineralocorticoid receptors. Hypertension 2008;52:1016-1018.
33 Di Zhang A, Cat AN, Soukaseum C, Escoubet B, Cherfa A, Messaoudi S, Delcayre C, Samuel JL, Jaisser F: Cross-talk between mineralocorticoid and angiotensin II signaling for cardiac remodeling. Hypertension 2008;52:1060-1067.

-34 Lesgards JF, Frayne IR, Comte B, Busseuil D, Rhéaume E, Tardif JC, Rosiers CD: Differential distribution of 4-hydroxynonenal adducts to sulfur and nitrogen residues in blood proteins as revealed using Raney nickel and gas chromatography-mass spectrometry. Free Radic Biol Med 2009;47:13751385.

35 Custodis F, Baumhäkel M, Schlimmer N, List F, Gensch C, Böhm M, Laufs U: Heart rate reduction by ivabradine reduces oxidative stress, improves endothelial function, and prevents atherosclerosis in apolipoprotein E-deficient mice. Circulation 2008;117: 2377-2387. 